\title{
La (no) panacea de "educación gratuita para todos"
}

Durante toda la campaña presidencia en contra del tema de la gratuidad universal en educación: una de las reformas fondo propuestas por la presidenta electa Michelle Bachelet. En este articulo queremos explicar por qué creemos que, antes superior debre gratuidad de la educación superior, debemos garantizar educación básico y medio Esto, no quiere decir que se deba "acabar" con el sector particular subvencionado, sino que deben existir nuevas reglas para el funcionamiento y financia miento de los colegios públicos, privados con subvención y para la carrera docente.

GRATUIDAD UNIVERSAL EN EDUCACIÖN SUPERIOR: EN QUÉ HAY QUE PENSAR La definición de las reglas del juego para el acceso a la educación superior y para su financiamiento no debe transformarse en una pollica de contencion, sino en una politica educativa que garantice igualdad que determine ambern án mecrismo que garantice la calidad de la educación y la oportunidad de la formación que se esta brindando. Por oportunidad nos referimos a las posibilidades laborales que van a tener los futuros egresados de las distintas carreras y niveles (técnico, profesional, universitario). Sin embargo, el poder ofrecer educacion "gratis para todos" en el contexto actual de la educación básica y medi lamentablemente no garantiza ni lo uno du to otro. Sino que perpetúa el estatus quo (siguen accediendo a educación superior con agregar un peso considerable sobre las arcas fiscales: estudiantes de ingresos altos estarán sobre representados en universidasos de calidad alta y estudiantes de ingresos bajos en universidades de baja calida Estamos entrampados por razones - Estamos entrampados por razones ajecuánto cada familia ha pagado o debe pagar Ya sabemos que la mera expansión del per se que estemos capacitando mejor a nuestros ciudadanos sino que al contrario, estamos conteniendo a los estudiantes en tres a cinco años más tarde los lanzará a un mercado laboral que no siempre reconoce los "titulos" y que además, discrimina segun la procedencia del egresado. Y aun cuando puede ser parte de un snobismo, e vo egrosamos de de ciertas profesiones

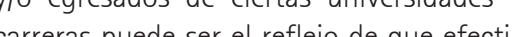
carras puede ser el rejo de que efectiestán formando profesionas o téch mpetentes.

mpdos en un tema de calidad y pertinencia de la formación universitaria que no garantiza a los alumnos (tanto los que pagan como los que no) el acceso a salidas laborales acordes a lo (teoricamente debieran haber adquirido. entonces, antes de pensar en poner plazos para hacer el sistema totalmente gratuito hay que atacar este otro flanco: el de la calidad, pertinencia y mecanismos de acceso al sistema de educación superior, lo que
MÁS CALIDAD Y MENOR SEGREGACIÓN NEL NIVEL BASICO Y MEDIO insticucionalidad en educación básica y media, resulta bastante 政 a existencia de colegios particulares sub-

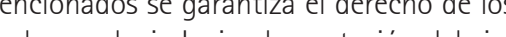
ama de subsid tas per capta (Vor del sisetablece con el propósito de promover proveedores públicos y pivados responsables del servicio educatiesperaba que los padres, ejerciendo elección del establecimiento generaran competencia entre colegios para captar estudiantes y asi obtener la subvención statal. Este mecanismo debiera haber genado presion para aumentar calidad en

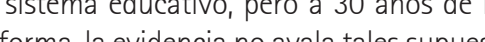
tos. Casi el $88 \%$ de los estudiantes de tos. Casi el $88 \%$ de los estudiantes de las escuelas que muestran una baja calidad historica media a través de SIMCE, per-

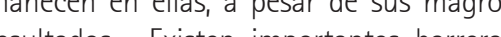
resultados. Existen importantes barreras

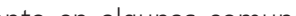
chelilas, por cuanto en algunas comunas enten, son inalcanzables para as familias en presencia de copagos de ma tricula. Muchos colegios también imponen pruebas de selección a los estudiantes. ducen de manera significativa los cupos pruebas estandarizadas claramente ponen 
incentivos a favor de estas prácticas.

nacionales e internacionales, Chile no sólo presenta magros resultados en lectura, matemáticas y ciencias, sino que también es uno de los paises en los que los resultanivel socioeconómico de los alumnos y de los colegios en los que estudian. Aprenden mayores recursos $y$ quintites de ingreso.

En esto no hay recetas mágicas todo

ha sido dicho (con mayor o menor énfasis) tanto por nosotras (en el articulo "Los desafios de mejorar la calidad ( y la equidad) de a educación básica y media en Chile"|como por otros académicos o think tanks. Para reducir el nivel de segregación de nuestro sistema educativo, hay que cambiar el régimen de financiamiento y efectivamente dotar Ministerio (o la agencia que se encargue de esto) de mayor poder de fiscalización. Para mejorar las competencias de nuestros lastudiantes se debe mejorar la calidad de los docentes y sus condiciones de trabajo. eficaces las prácticas pedagógicas y los métodos de enseñanza y por sobre todos me-

anza, y por sobre todo, pon igual calidad educativa para todos los estudiantes que asisten a escuelas con financiamiento público; sean estos de gestión privada o municipal, de sectores rurales urbanos, de distintos nivel socioeconómco, u otros. Estos dos últimos puntos son aspectos esenciales y estratégicos para incrementar la calidad e igualdad de oportunidades en cada escuela del sistema educativo. El proceso de enseñanza y aprendizaje en el aula es por lejos, el factor de mayor impacto e incidencia en los aprendizajes sarrollo peno eintegral de los niños, niñasy buenes. Es necesario fortalecer en los estudiantes el pensamiento critico autónomo a creatividad. La dinámica e interacción social y pedagógica que caracteriza y enmarca la enseñanza y el aprendizaje en la sala de clases resulta esencial para aquello que ninos $\mathrm{y}$ jóvenes aprenden o dejan de aprender durante su proceso escolar. Mejorar calidad del proceso que alli ocurre, implica fortalecer la formación y profesionalización de los docentes, junto con la gestion y condiciones institucionales para lograr el éxito tal labor.
SOBRE EL RÉGIMEN DE FINANCIAMIENTO DEL SISTEMA sistema aspectos netamente técnicos, demanda, y al igual que en muchos otros totalmente obsoleto. Se comienza con una tener un mecanismo público y transparensubvención escolar básica (subvención te para selección de alumnos, basados por regular), y luego hay un montón de "adi- ejemplo en loterías (sorteos). Sólo se poestán sujetas a reglas y especiales, que drian permitir instrumentos de selección control distintos parece del todo neesario asumnos pos aún hestuvieran en el conas de transitar hacia un mecanismo de financia- con el objeto de favorecer la integración de miento que refleje la estructura de costos las familias en una sola comunidad escocuasi-fija que enfrenta un colegio tipico lar En esta dirección, se ha avanzado con a para su funcionamiento, asi como también introducción de la ley SEP, ya que prohibe las características propias de las familias y la selección de estudiantes en los primeros de los alumnos que atiende. En particular años de básica en aquellos colegios que recualquier esquema de financiamiento de- ciben esta subvención adicional a la regular biera tener componentes que sean fijos, (vouchers). Prohibe igualmente que se les otros que dependan de asistencia promedio cobre un monto adicional (financiamiente anual y den cuenta de condiciones parti- compartido) a las familias de estudiantes culares del colegio: condición de ruralidad, vulnerables por los cuales se recibe esta sostenedor (en red o inla comuna, tipo de Subvención Preferencial. La medida sin emsostenedor (en red o independiente), entre bargo, no ha sido lo suficientemente estu-

Complementario a este cambio, deben heterogeneidad de la población escolar en ave especificas son no sólo necesaias sino cios de problemas de discriminción de los que indispensables para terminar con la estudiantes vulnerables en escuelos de les

segmentación del sistema. Suena cuerdo y socioeconómico más alto (Román, Perticará justo que en primer lugar se exija que todo colegio que quiera recibir subsidio público deje de percibir recursos mediante financiamiento compartido (via cobro adicional mensual a las familias). Si el colegio recibe fondos públicos, no puede pedir además aportes directos a las familialpara uniformes o utiles escolares de marca, por ejeme. el actual sistema de financiamiento comescolar, dado que al interior de un sistema escolar, dado que al interior de un sistema desigualmente y que las escuelas bajo esta molalida concentran a estudistes consimilar capacidad de pago y con ello se levanta nuevas barreras de exclusión o selección por variables económicas (Valenzuela et at. 2009; Elacqua, 2012, Elacqua et al., 2013. Entendemos también que la eliminación del nanciamiento compartido es algo que de hacerse gradual y planificadamente. Por otra parte, los colegios que reciben subvention estatal no debieran bajo ningún en ninguno de sus niveles y grados, como tampoco aplicar criterios de desempeño Selman 2013)

Las nuevas reglas de financiamiento y estión de la matrícula, deben ir acompate una regulación "sana" pero efec(tive "srocedimiento administrativo para . financiamiento a una sola, claramente va en esta dirección.

\section{LA (OLVIDADA) CARRERA DOCENTE.}

No es posible que los niños aprendan si no Noes posibleque los nintosaprendan Necesitamos tener a los mejores profesors mando de las aulas: a los más competentes, comprometidos y apasionados con a labor docente. Y el tener profesores capaces tiene que ver con tener buenos alumran ser profesores; con generar se mantengan en labores lectivas, que se sientan comprometidos y que les sea atractive ir compenar en esculas de contextos mas complejos, aislados o dificilies. Y estos tha "sano" tiene que ver con que cualtos sea centralizado en una sola agencia de incentivos para que los buenos profesores también con condiciones de trabajo, desde las horas de clase presenciales, hasta posibilidades de capacitación, empoderamiento del docente, y tambien mejoras sustancial en la gestión de los colegios.

Tres propuestas especificas: 1. Incentivos a estudiar carreras de profesorado. Es esencial atraer a estudiantes mativados y con aptitudes. Es complejo esPSU, pero claromente deben establecerse ciertos estándares minimos para poder acceder a estas carreras. Y lo primordial hay que definir mecanismos de habilitació para quienes pueden ejercer la docencia. $S$ propone entonces, continuar incentivando la matrícula de jóvenes con aptitudes, pero con un mecanismo mixto para acceder tanto a un cupo en una carrera de pedagogía como a financiamiento público que contemple PSU, promedio en la enseñanza media, ranking de notas, y entrevistas con los directores de carrera. Asi mismo, todos aquellos alumnos que reciban financia miento público para financiar sus carreras deberán trabajar a menos tres años en $\mathrm{CO}^{-}$ legios subvencionados con preferenci establecimientos de alta vulnerabilidad. 2 Calidad de las carreras de pedagog Relacionado con el punto anterior, es imperioso mejorar la formación de los estudiantes de pedagogía. La prueba INICIA revela una alta heterogeneidad entre las Universidades tradicionales y las privadas. No claro si la heterogeneidad es producto de alumnos con puntos de inicio distintos producto de buenos y malos programas de educación. Con todo, lo relevante para sistema es tener buenos profesores. No puede hacer politica social, entregando un univeritaios vulnerabes para que luego estos repliquen sus mismas falencias dentro de su mismo estrato socioeconómico. Si queremos darle la oportunidad a jóvenes con menos aptitudes de ser profesores (porque es su vocacion. tendremos entonces que garantizar un proceso formativo que los ubique en un nivel de conocimientos similar a sus pares más aventajados. En el largo plazo, si lograramos mejoras en la educación básica y media subvencionada debièramos tener la cancha mas nivelad (por nivel socioeconómico) a la entrada a que quien no tiene las aptitudes no puede pararse en un aula de clases. debe ser un examen nacional unico y obligatorio que contemple desde conocimientos disciplinars hasta practicas pedagógique no cump con el pungun egresado este examen podrá ejercer laje minimo en ser contratas por riganos (munipios u otros) para cumplir bores de enseñanza en sus escuelas.

Salarios y condiciones de empleo de los docentes. Luego de atraer a buenos alumnos para estudiar pedagogía, y lograr "producir" buenos profesionales, es importante retenerlos. Actualmente, un docente do inicia su carrera gana $75 \%$ de lo que gana un trabajador a tiempo completo. el salario de entrada (entre 400-600 mil pesos), premiando incluso a aquellos docentes que opten por colegios más vulnerables 0 en zonas más aisladas. La escala de salarios debe contemplar asignaciones por cargos entre los distintos niveles de enseñanza Cualquier pago por incentivo o mérito debe darse directamente a los profesores y no asignarse un fondo a los sostenedores, sobre el que la fiscalización de los destinos de los dineros es dificil de ejecutar.

Se deben también aumentar de manera importante los premios por desempeño unificando las instancias y los fondos que existen para acceder a estos pagos. Estos premios permiten a los docentes avanzar en la escala salarial, sin distinciones con respecto a edad y/o años de experiencia aboral. Debe haber un unico examen para obtener estos bonos al mérito, que debe folio, sino lo evaluacín del donte en una

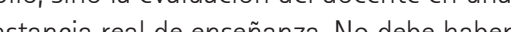
ningún requisito sobre quién puede tomar el examen y acceder al beneficio monetario como por ejemplo cantidad de horas semanales de clase y/o dependencia del establesitar hacia una carga de horas lectiva mâs En te con los estandares internacionales. lectivas, cuando paises desarrollados están en torno a 50-60\%, lo que además se transforma en una muy buena excusa para postergar la planificación y organización de la enseñanza, factores esenciales pa mejorar el trabajo y resultados del aula. Gradualmente, debemos reestructurar las feriones de producción de los colegios

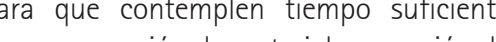
controles, y capacitación

Podemos concluir ex (a) ción pertinente y de calidad en el nivel báscoy medio. Ninguna politica o instrumento que pueda aplicarse al nivel de educación superior generará equidad a menos que podamos nivelar las habilidades de los niños independientemente de su nivel socioecogio de procedencia.

En el intertanto, se debe comenzar trabajar en mejorar el sistema de asegura . 政 vendo a reproducir inequidades.

Román, Marcela y Perticara, Marcela (2012. calidad en Chile"." Estudios de Economia 39 (2), pp. 59-167, Diciembre.

\section{洒}

e- -mail: jrodrigu@uahurtado. 